\title{
Specific components of face perception in the human fusiform gyrus studied by tomographic estimates of magnetoencephalographic signals: a tool for the evaluation of non-verbal communication in psychosomatic paradigms
}

\author{
Yuka Okazaki*1,2 and Andreas A Ioannides ${ }^{1,2}$
}

Address: ${ }^{1}$ Department of Brain Science and Engineering, Graduate School of Life Science and Systems Engineering, Kyushu Institute of Technology, Kitakyushu-shi, Japan and 2Laboratory for Human Brain Dynamics, Brain Science Institute (BSI), RIKEN, Wako-shi, Japan

Email: Yuka Okazaki* - yuka@brain.riken.jp; Andreas A Ioannides - ioannides@postman.riken.jp

* Corresponding author

Published: 4 December 2007

BioPsychoSocial Medicine 2007, I:23 doi:I0.1 186/I75 I-0759-1-23

This article is available from: http://www.bpsmedicine.com/content/l/l/23

(c) 2007 Okazaki and loannides; licensee BioMed Central Ltd.

This is an Open Access article distributed under the terms of the Creative Commons Attribution License (http://creativecommons.org/licenses/by/2.0), which permits unrestricted use, distribution, and reproduction in any medium, provided the original work is properly cited.
Received: 14 November 2007

Accepted: 4 December 2007

\begin{abstract}
Aims: The aim of this study was to determine the specific spatiotemporal activation patterns of face perception in the fusiform gyrus (FG). The FG is a key area in the specialized brain system that makes possible the recognition of face with ease and speed in our daily life. Characterization of FG response provides a quantitative method for evaluating the fundamental functions that contribute to non-verbal communication in various psychosomatic paradigms.
\end{abstract}

Methods: The MEG signal was recorded during passive visual stimulus presentation with three stimulus types - Faces, Hands and Shoes. The stimuli were presented separately to the central and peripheral visual fields. We performed statistical parametric mapping (SPM) analysis of tomographic estimates of activity to compare activity between a pre- and post-stimulus period in the same object (baseline test), and activity between objects (active test). The time course of regional activation curves was analyzed for each stimulus condition.

Results: The SPM baseline test revealed a response to each stimulus type, which was very compact at the initial segment of main $\mathrm{M}_{\mathrm{FG}}$ 170. For hands and shoes the area of significant change remains compact. For faces the area expanded widely within a few milliseconds and its boundaries engulfed the other object areas. The active test demonstrated that activity for faces was significantly larger than the activity for hands. The same face specific compact area as in the baseline test was identified, and then again expanded widely. For each stimulus type and presentation in each one of the visual fields locations, the analysis of the time course of $F G$ activity identified three components in the $F G: M_{F G} 100, M_{F G} 170$, and $M_{F G} 200$ - all showed preference for faces.

Conclusion: Early compact face-specific activity in the FG expands widely along the occipitoventral brain within a few milliseconds. The significant difference between faces and the other object stimuli in $\mathrm{M}_{\mathrm{FG}} \mathrm{I} 00$ shows that processing of faces is already differentiated from processing of other objects within 100 ms. Standardization of the three face-specific MEG components could have diagnostic value for the integrity of the initial process of non-verbal communication in various psychosomatic paradigms. 


\section{Findings}

Faces constitute perhaps the most important stimuli in social interactions. Facial expression is one of the most informative non-verbal cues and it is analyzed soon after the initial process for non-verbal communication related to face perception is completed [1]. It is well-known that face perception is under the great influence of attention, emotional states and various neuro-endocrine conditions [2]. A quantitative analysis of the neural process of face perception may provide a tool to evaluate the fundamental functions of the initial step of non-verbal communication, which is impaired in certain psychosomatic patients. However, the results obtained so far are unclear about how face specificity develops in space and time.

Many fMRI studies have identified activations elicited by faces in compact ventral parts of the regions, in particular within the fusiform gyrus (FG) [3-5]. Other fMRI studies demonstrated that when information from a number of $\mathrm{fMRI}$ voxels is combined, a number of different visual stimuli, not just faces, elicit category specific responses [68]. Since the fMRI signal relies on hemodynamics, these results relate to slow processes, with a characteristic timescale of seconds. Intracranial recordings provide information with high temporal resolution and spatial accuracy, but only at the locations dictated by clinical requirements. Such invasive measurements have identified a face-specific N200 that spread over ventral and temporal aspects of the brain [9]. MEG studies claimed face specificresponses in the FG for the M170 component [10,11]. A few studies have reported early face induced responses within $100 \mathrm{~ms}$, some in early visual areas [10,12], while others were in the FG [13]. The last study did not use nonface control stimuli, so it could only demonstrate that face stimuli elicit early activity in FG, but could not determine whether this component was face-specific. What is therefore needed is accurate tomographic localization and precise timing in the measurement of face and non-face stimuli within the same experiment.

Seven, healthy right-handed Caucasian male subjects (mean age, $30.0 \pm 5.0$ years) gave informed, written consent, after the protocol was explained to them. The MEG protocol was approved by the Research Ethics Committee of RIKEN.

We used grayscale images of faces, hands and shoes. Shoe images were provided courtesy of the website [14]. Each stimulus was presented for $300 \mathrm{~ms}$ in one of five locations, either in the center or at $10.7^{\circ}$ eccentricity from fixation across the diagonal. In each run, stimulus was presented five times in three of the five locations, alternating the choices to equally cover all five locations in the different runs. In central presentations, faces, hands, and shoes were presented at sizes of $5.5 \times 4.1^{\circ}, 4.5 \times 3.6^{\circ}$, and
$4.8 \times 3.5^{\circ}$ respectively. In the periphery, images were 8.2 $\times 6.1^{\circ}, 6.7 \times 5.3^{\circ}$ and $7.1 \times 5.2^{\circ}$ in size respectively. The subject's task was to fixate on the central cross and to respond to the subtle change in its color with a quick button press.

Magnetic fields were measured with the MEG systems (Omega 151, CTF Systems Inc., Vancouver, B.C., Canada) in a magnetically shielded room (MSR). The Presentation software (Neurobehavioral Systems, Inc., Albany, CA) controlled a DLP projector with a $96 \mathrm{~Hz}$ refresh rate (HL8000Dsx+, NEC Viewtechnology Ltd., Tokyo, Japan) located outside the MSR. The exact onset time of each stimulus was determined by luminance detection with a photodiode on the screen. The EOG and ECG were simultaneously recorded and trials with eye movements or blinks exceeding $50 \mu \mathrm{V}$ EOG signal change during the stimulus presentation period were discarded. Remaining artifacts were identified by strong ICA components correlated with either EOG or ECG and were removed. The signals from all channels were digitized at a sampling-rate of $625 \mathrm{~Hz}$. The MEG signal was filtered with a bandwidth of $3-200 \mathrm{~Hz}$ and with notches at $50 \mathrm{~Hz}$ and its harmonics to eliminate power-line noise. The MEG sensors were determined relative to the individual subject MRI images for each run by the localization of fiduciary coils and our in-house co-registration procedure [15]. Magnetic field tomography (MFT) $[16,17]$ was applied to each time slice of data (every 1.6 milliseconds apart) to extract independently tomographic estimates of neuronal activity.

For each subject, we performed voxel-by-voxel statistical parametric mapping (SPM) analysis by comparing the sample distribution for modulus of the MFT solutions, separately for each stimulus and VF. Two types of SPM analysis were performed, with Bonferroni-correction applied in each case to account for Type I errors due to multiple voxel comparisons. In the active test SPMs were produced by comparing the samples within a latency window (width $=19.2 \mathrm{~ms}$ ) between two conditions. In the baseline test the comparison was between samples consisting of one sample from every three runs in the post-stimulus period and random samples from the pre-stimulus period ( $-250 \mathrm{~ms}$ to $-50 \mathrm{~ms})$. More details on SPM analysis can be found elsewhere [18].

To define regions of interest (ROIs) for the left and right FG in each subject, we used anatomical criteria - the collateral sulcus and the temporal occipital sulcus, and the SPM results of baseline test. The ROIs for central and contralateral peripheral presentations were defined independently of each other. We used circular statistics [19,20] to define the dominant direction of the MFT current density elicited by face stimuli in the $120 \mathrm{~ms}$ to $180 \mathrm{~ms}$ range inside the given ROI with a radius of $10 \mathrm{~mm}$. 
After ROI definition, a regional activation curve (RAC) was calculated for each stimulus condition at every timeslice by projection of the current density vector onto the dominant direction. Momentary amplitudes of the RAC were analyzed using ANOVA, following the same conceptual steps as for the SPM analysis, but with a 4.8 ms running window stepped every $1.6 \mathrm{~ms}$. In the RAC active test, an ANOVA was performed with Stimulus type (Faces, Hands, Shoes), Hemisphere (left, right), and VF (upper, lower) as fixed factors, and Subject (seven subjects) as a random factor. Period (pre-stimulus, post-stimulus) was added as an additional fixed factor in the RAC baseline test.

Highly significant SPM foci for each object were intermittently identified from about $90 \mathrm{~ms}$ to $230 \mathrm{~ms}$ in FG. Face stimuli elicited activity within $100 \mathrm{~ms}$. The contours in Figures $1 \mathrm{~A}$ and $1 \mathrm{~B}$ show the common significant FG activations across subjects around $130-150 \mathrm{~ms}$ for the baseline $(p<0.05)$ and active $(p<0.005)$ tests. For the baseline test the contours for Faces expanded within a few milliseconds and engulfed the other object area. Activity for nonface stimuli were consistently compact compared to face. The active test indicated significantly higher activity for Faces than Hands, over a wide area that included the area activated by Hands in the baseline test.

The subject specific ROIs as displayed in figure 1C were used to compute RAC. Figure 1D shows the grand averaged RAC across subjects for each stimulus type, separately for center and periphery. The RAC baseline test identified statistically significant FG activation across subjects in three periods. For central presentation these were at $71.0-121.0 \mathrm{~ms}(F(1,6)=47.6, p<0.05), 135.0-186.0$ $\mathrm{ms}(F(1,6)=22.4, p<0.05)$, and $204.0-263.0 \mathrm{~ms}(F(1$, $6)=20.2, p<0.05)$. For the peripheral presentation, significant activation periods were at $65.0-113.0 \mathrm{~ms}(F(1,6)$ $=30.2, p<0.05), 127.0-177.0 \mathrm{~ms}(F(1,6)=45.3, p<$ $0.05)$, and 207.0-263.0 ms $(F(1,6)=35.3, p<0.05)$.

We performed a post-hoc test (Tukey's method) for the most significant main effects of stimuli at the three identified peaks. No main effect of Hemisphere or Stimulus type $\times$ Hemisphere interaction was found. In the expected face selectivity at $\mathrm{M}_{\mathrm{FG}} 170$, the amplitude for the central presentation of face stimuli became significantly stronger than the other objects at $135.0 \mathrm{~ms}$ with a main effect of Stimulus type according to ANOVA $(F(2,12)=8.76, p<$ $0.005)$, and showed an amplitude peak at $153.0 \mathrm{~ms}$. Similarly, peripheral presentation showed a stronger response to faces at $126.0 \mathrm{~ms}(F(2,12)=7.11, p<0.01)$, with an amplitude peak at $150.0 \mathrm{~ms}$. Subsequent significant $\left(\mathrm{M}_{\mathrm{FG}} 200\right)$ differences were found at $207.0 \mathrm{~ms}$ for the central presentations $(F(2,12)=4.97, p<0.05)$ and $246.0 \mathrm{~ms}$ for peripheral presentations $(F(2,12)=13.81, p<0.001)$.
The analysis revealed that the response within $100 \mathrm{~ms}$ $\left(\mathrm{M}_{\mathrm{FG}} 100\right)$ was also stronger for face stimuli compared to other objects, peaking at $73.0 \mathrm{~ms}$ (range, $63.0-81.0 \mathrm{~ms}$ ), and $65.0 \mathrm{~ms}$ (range, $60.0-81.0 \mathrm{~ms}$ ), for central $(F(2,12)$ $=13.15, p<0.001)$ and peripheral presentations $(F(2,12)$ $=11.14, p<0.05)$, respectively.

Our results resolve the apparent contradictions in previous fMRI and intracranial studies regarding object-selective responses in the ventral visual stream and add a critical temporal dimension to the analysis. In short, we found a compact response during the initial segment of the main component $\left(\mathrm{M}_{\mathrm{FG}} 170\right)$. Compared to the baseline, the area of higher activity for hands and shoes remained compact, but the area for faces expanded widely and engulfed (just within its borders) the areas for hands and shoes. Direct comparison between faces and hands showed increased activity for faces, with a compact area identical to the early compact area of the face baseline test. This area expanded widely in the next few milliseconds. Thus our results show the compact face-specificity in the FG reported in some fMRI studies [3-5] during the early segment of each object-specific response. A few milliseconds later the compact face-specific activation in the FG expands, widely reproducing the pattern seen along the ventro-temporal cortex in other fMRI studies [6-8] and in studies with intracranial recording [9].

The time course for FG showed three components. The strongest peaked around $170\left(\mathrm{M}_{\mathrm{FG}} 170\right)$ in agreement with many other studies $[10,21,22]$. An earlier component peaked within $100 \mathrm{~ms}\left(\mathrm{M}_{\mathrm{FG}} 100\right)$, and a later one after 200 $\mathrm{ms}\left(\mathrm{M}_{\mathrm{FG}} 200\right)$, again in agreement with previous studies $[12,13]$. Analysis of these time courses demonstrated a clear bias toward face stimuli for all three components. The face-selectivity of the early FG activity within $100 \mathrm{~ms}$, demonstrated for the first time in our study, is particularly relevant to models attempting to explain how facial identity is processed with speed and accuracy.

In the present study, we found the three specific components of face perception in the FG area. Preliminary results from further analysis of our data suggest that the later components are modulated by attention. Detail analyses of $\mathrm{M}_{\mathrm{FG}} 100, \mathrm{M}_{\mathrm{FG}} 170$ and $\mathrm{M}_{\mathrm{FG}} 200$ in psychosomatic patients may reveal initial processes of face perception in non-verbal communication that are impaired in certain psychosomatic patients, especially patients with alexithymia $[23,24]$.

\section{List of abbreviations}

ECG: Electrocardiogram;

EOG: Electrooculogram; 

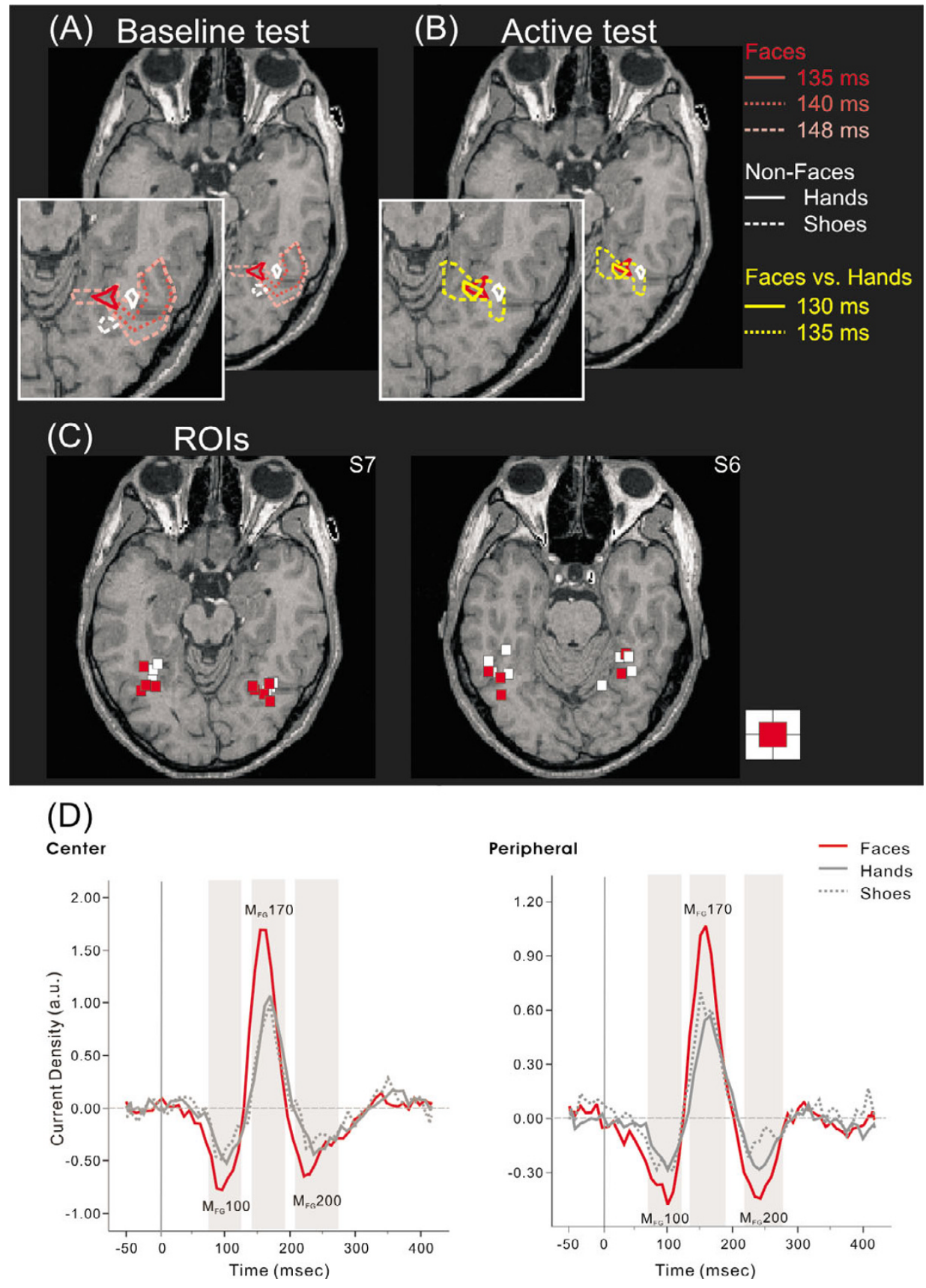

Figure I

(A) Common activations from SPM baseline tests for central presentation. The contours delineate areas where the activity during the active period is higher $(p<0.05)$ than the baseline for at least 5 out of 7 subjects. Separate contours show the results for face (gradation red - different timing), hand (white solid) and shoe (white dash) stimuli. (B) Results displayed in the same format as in (A) but for the active test with contours delineating areas where the activity for face stimuli is higher $(P<$ 0.005 ) than hands in all seven subjects (yellow). The baseline result for faces at $135 \mathrm{~ms}$ is copied from (A) and it can just be distinguished from the active test at I30 ms. (C) ROI centers (identified by the baseline test of) for all subjects, transformed via common Talairach coordinates to the space of the MRI display. Red and white squares mark the ROI centers of individual subjects for central and peripheral presentations. (D) The regional activation curves (RAC). RAC are averaged across subjects in the left and right FG for central and peripheral visual field presentation. The gray, shaded areas mark periods with activity higher than baseline $(-250 \mathrm{~ms}--50 \mathrm{~ms})$. For both center and periphery, three components can be clearly seen: $M_{\mathrm{FG}} 100$, $\mathrm{M}_{\mathrm{FG}} 170$, and $\mathrm{M}_{\mathrm{FG}} 200$. 
fMRI: Functional magnetic resonance imaging;

ICA: Independent component analysis;

MEG: Magnetoencephalography.

\section{Authors' contributions}

YO carried out the MEG experiment and performed analysis. AAI conceived of the study and participated in its design and coordination. The manuscript was drafted by both authors.

\section{Acknowledgements}

We thank Arman Abrahamyan and Catherine Stevens for their contribution in the preparation of the stimuli and the design of the experiment. We also thank Kenji Haruhana for his help in running the experiments, Vahe Poghosyan for his help in data analysis and Masaki Maruyama for his valuable discussion and advice in data analysis.

\section{References}

I. Kubota JT, Ito TA: Multiple Cues in Social Perception: The Time Course of Processing Race and Facial Expression. J Exp Soc Psychol 2007, 43:738-752.

2. Barton J], Hefter RL, Cherkasova MV, Manoach DS: Investigations of face expertise in the social developmental disorders. Neurology 2007, 69:860-870.

3. Kanwisher N, McDermott J, Chun MM: The fusiform face area: a module in human extrastriate cortex specialized for face perception. J Neurosci 1997, I 7:4302-43 I I.

4. Schwarzlose RF, Baker Cl, Kanwisher N: Separate face and body selectivity on the fusiform gyrus. I Neurosci 2005, 25: II055-11059.

5. Hadjikhani N, de Gelder B: Neural basis of prosopagnosia: an fMRI study. Hum Brain Mapp 2002, 16:176-I82.

6. Haxby JV, Gobbini MI, Furey ML, Ishai A, Schouten JL, Pietrini P: Distributed and overlapping representations of faces and objects in ventral temporal cortex. Science 200I, 293:2425-2430.

7. Ishai A, Ungerleider LG, Haxby JV: Distributed neural systems for the generation of visual images. Neuron 2000, 28:979-990.

8. Ishai A, Ungerleider LG, Martin A, Schouten JL, Haxby JV: Distributed representation of objects in the human ventral visual pathway. Proc Natl Acad Sci U S A 1 999, 96:9379-9384.

9. Allison T, Puce A, Spencer DD, McCarthy G: Electrophysiological studies of human face perception. I: Potentials generated in occipitotemporal cortex by face and non-face stimuli. Cereb Cortex 1999, 9:4I5-430.

10. Watanabe S, Kakigi R, Koyama S, Kirino E: Human face perception traced by magneto- and electro-encephalography. Brain Res Cogn Brain Res 1999, 8: I25-142.

II. Liu L, loannides AA, Streit M: Single trial analysis of neurophysiological correlates of the recognition of complex objects and facial expressions of emotion. Brain Topogr 1999, I I:29|-303.

12. Itier RJ, Herdman AT, George N, Cheyne D, Taylor MJ: Inversion and contrast-reversal effects on face processing assessed by MEG. Brain Res 2006.

13. Liu L, loannides AA: Spatiotemporal dynamics and connectivity pattern differences between centrally and peripherally presented faces. Neuroimage 2006, 31:1726-1740.

14. Shoes.com: [http://www.shoes.com].

15. Hironaga N, loannides AA: Accurate co-registration for MEG reconstructions. Edited by: Nowak $\mathrm{H}$, Haueisen J, GieBler $\mathrm{F}$ and Huonker R. Berlin, Verlag; 2002:93I-933.

16. Ioannides AA, Bolton JPR, Clarke CJS: Continuous Probabilistic Solutions to the Biomagnetic Inverse Problem. Inverse Problems 1990, 6:523-542.

17. Taylor JG, loannides AA, Muller-Gartner HW: Mathematical analysis of lead field expansions. IEEE Trans Med Imaging 1999, 18:151-163.
18. Poghosyan $\mathrm{V}$, Shibata $\mathrm{T}$, loannides $\mathrm{AA}$ : Effects of attention and arousal on early responses in striate cortex. Eur J Neurosci 2005, 22:225-234.

19. Fisher NI: Statistical analysis of circular data NY, Cambridge University Press; 1993.

20. loannides AA, Fenwick PB, Liu L: Widely distributed magnetoencephalography spikes related to the planning and execution of human saccades. J Neurosci 2005, 25:7950-7967.

21. Streit M, loannides AA, Liu L, Wolwer W, Dammers J, Gross J, Gaebel W, Muller-Gartner HW: Neurophysiological correlates of the recognition of facial expressions of emotion as revealed by magnetoencephalography. Brain Res Cogn Brain Res |999, 7:48|-49|.

22. Halgren E, Raij T, Marinkovic K, Jousmaki V, Hari R: Cognitive response profile of the human fusiform face area as determined by MEG. Cereb Cortex 2000, 10:69-8I.

23. Schultz RT: Developmental deficits in social perception in autism: the role of the amygdala and fusiform face area. Int J Dev Neurosci 2005, 23:125-141.

24. Schafer R, Popp K, Jorgens S, Lindenberg R, Franz M, Seitz RJ: Alexithymia-like disorder in right anterior cingulate infarction. Neurocase 2007, 13:20I-208.
Publish with Biomed Central and every scientist can read your work free of charge

"BioMed Central will be the most significant development for disseminating the results of biomedical research in our lifetime. "

Sir Paul Nurse, Cancer Research UK

Your research papers will be:

- available free of charge to the entire biomedical community

- peer reviewed and published immediately upon acceptance

- cited in PubMed and archived on PubMed Central

- yours - you keep the copyright 